\title{
GENERIC SUBMANIFOLDS OF COMPLEX PROJECTIVE SPACES WITH PARALLEL MEAN CURVATURE VECTOR
}

\author{
Dedicated to Professor Shigeru Ishihara on his sixtieth birthday
}

\author{
By U-Hang Ki, Jin Suk Pak and Young Ho Kim
}

A submanifold $M$ of a Kaehlerian manifold $\tilde{M}$ is called a generic submanifold (an anti-holomorphic submanifold) if the normal space $N_{P}(M)$ of $M$ at any point $P \in M$ is always mapped into the tangent space $T_{P}(M)$ under the action of the almost complex structure tensor $F$ of the ambient manifold, that is, $F N_{P}(M) \subset$ $T_{P}(M)$ for all $P \in M$ (see [4], [9], [10] and [12]). The typical examples of generic submanifolds are real hypersurfaces of a Kaehlerian manifold. So many authors, for example, Kon [12], Okumura [9], Pak [9] and Yano [12] etc., have studied generic submanifolds of a Kaehlerian manifold by using the method of Riemannian fibre bundles and developed this method of Lawson [2], Maeda [5] or Okumura [8] extensively for real hypersurfaces.

In particular, two of the present authors [4] have studied generic submantfolds with parallel mean curvature vector of an even-dimensional Euclidean space under the condition that the $f$-structure induced on $M$ is normal (see section 2).

The purpose of the present paper is to characterize generic submanifolds of complex projective space $C P^{m}$.

In $\S 1$, we investigate fundamental properties and structure equations for generic submanifolds immersed in a complex projective space $C P^{m}$. And we find the condition that the $f$-structure induced on $M$ is normal.

In $\S 2$, we recall the theory of fibrations and some relations between the second fundamental tensor of $M$ in $C P^{m}$ and that of $\bar{M}=\tilde{\pi}^{-1}(M)$ in $S^{2 m+1}$, and then establish some equations for the connections in the normal bundles of $M$ and of $\bar{M}$, where $\tilde{\pi}$ is the projection induced from the Hopf-fibrations $S^{1} \rightarrow S^{2 m+1} \rightarrow C P^{m}$.

In the last $\S 3$, we characterize generic submanifolds of a complex projective space $C P^{m}$ by the method of Riemannian fibration. In characterizing the submanifolds, we shall use the following theorem:

THEOREM A ([11]). Let $M$ be a complete n-dimensional submanifold of $S^{m}$ with flat normal connection. If the second fundamental form of $M$ is parallel, then $M$ is a small sphere, a great sphere or a pythagorean product of a certain number of spheres. Moreover, if $M$ is of essential codimension $m-n$, then $M$ is a pythagorean product of the form

Received December 15, 1979 


$$
S^{p_{1}}\left(r_{1}\right) \times \cdots \times S^{p_{N}}\left(r_{N}\right), r_{1}{ }^{2}+\cdots+r_{N}{ }^{2}=1, N=m-n+1,
$$

or a pythagorean product of the form

$$
S^{p_{1}}\left(r_{1}\right) \times \cdots \times S^{p_{N^{\prime}}}\left(r_{N^{\prime}}\right) \subset S^{m-1}(r) \subset S^{m}, r_{1}{ }^{2}+\cdots+r_{N^{\prime}}{ }^{2}=r^{2}<1, N^{\prime}=m-n .
$$

Manifolds, submanifolds, geometric objects and mappings we discuss in this paper are assumed to be differentiable and of $C^{\infty}$, We use in the present paper the systems of indices as follows:

$$
\begin{gathered}
\kappa, \mu, \nu, \lambda=1,2, \cdots, 2 m+1 ; h, \imath, \jmath, k=1,2, \cdots, 2 m, \\
\alpha, \beta, \gamma, \delta, \varepsilon=1,2, \cdots, n+1 ; a, b, c, d, e=1,2, \cdots, n, \\
u, v, w, x, y, z=1,2, \cdots, p, n+p=2 m .
\end{gathered}
$$

The summation convention will be used with respect to those systems of indices.

\section{$\S 1$. Generic submanifolds of Kaehlerian manifolds.}

Let $\tilde{M}$ be a $2 m$-dimensional Kaehlerian manifold covered by a system of coordinate neighborhoods $\left\{\tilde{U} ; y^{h}\right\}$ and denote by $g_{j i}$ components of the Hermitian metric tensor and by $F_{j}{ }^{h}$ those of the almost complex structure of $\tilde{M}$. Then we have

$$
\begin{aligned}
& F_{\jmath}{ }^{t} F_{t}{ }^{h}=-\delta_{\jmath}^{h}, \\
& F_{\jmath}{ }^{t} F_{\imath}{ }^{s} g_{t s}=g_{j \imath},
\end{aligned}
$$

$\delta_{\jmath}^{h}$ being the Kronecker delta.

And denoting by $\nabla$, the operator of covariant differentiation with respect to $g_{j i}$, we get

$$
\nabla_{j} F_{\imath}{ }^{h}=0 .
$$

Let $M$ be an $n$-dimensional Riemannian manifold covered by a system of coordinate neighborhoods $\left\{U ; x^{a}\right\}$ and immersed isometrically in $M$ by the immersion $\imath: M \rightarrow \tilde{M}$. We identify $i(M)$ with $M$ itself and represent the immersion $\imath: M \rightarrow \tilde{M}$ by

$$
y^{h}=y^{h}\left(x^{a}\right)
$$

We put

$$
B_{b}{ }^{h}=\partial_{b} y^{h}, \quad \partial_{b}=\partial / \partial x^{b}
$$

and denote by $C_{x}{ }^{h}$ mutually orthogonal unit normals to $M$. Then denoting by $g_{c b}$ the fundamental metric tensor of $M$, we have $g_{c b}=g_{j i} B_{c}{ }^{3} B_{b}{ }^{2}$ since the immersion is isometric. Therefore, denoting by $\nabla_{c}$ the operator of van der Waerden- 
Bortolotti covariant differentiation with respect to $g_{c b}$, equations of Gauss and Weingarten for $M$ are given by

$$
\begin{aligned}
& \nabla_{c} B_{b}{ }^{h}=h_{c b}{ }^{x} C_{x}{ }^{h}, \\
& \nabla_{c} C_{x}{ }^{h}=-h_{c}{ }^{a}{ }_{x} B_{a}{ }^{h}
\end{aligned}
$$

respectively, where $h_{c b}{ }^{x}$ are the second fundamental tensors with respect to the normals $C_{x}{ }^{h}$ and $h_{c}{ }^{a}{ }_{x}=h_{c b x} h^{b a}=h_{c b}{ }^{y} g^{b a} g_{y x}, g_{y x}=C_{y}{ }^{j} C_{x}{ }^{2} g_{\jmath i}$ being the metric tensor of the normal bundle of $M$ and $\left(g^{b a}\right)=\left(g_{b a}\right)^{-1}$.

Equations of Gauss, Codazzi and Ricci are respectively given by

$$
\begin{gathered}
K_{d c b}{ }^{a}=K_{k j i}{ }^{h} B_{d c b h}^{k j a}+h_{d}{ }^{a}{ }_{x} h_{c b}{ }^{x}-h_{c}{ }^{a}{ }_{x} h_{d b}{ }^{x}, \\
K_{k j i}{ }^{h} B_{d c b}^{k j i} C^{x}{ }_{h}=\nabla_{d} h_{c b}{ }^{x}-\nabla_{c} h_{d b}{ }^{x}, \\
K_{d c y}{ }^{x}=K_{k j i}{ }^{h} B_{d c}^{k j} C_{y}{ }^{2} C^{x}{ }_{h}+h_{d e}{ }^{x} h_{c}{ }^{e} y-h_{c e}{ }^{x} h_{d}{ }^{e}{ }_{y},
\end{gathered}
$$

where $B_{d c b h}^{k j a}=B_{d}{ }^{k} B_{c}{ }^{j} B_{b}{ }^{2} B^{a}{ }_{h}, \quad B_{d c b}^{k j i}=B_{d}{ }^{k} B_{c}{ }^{j} B_{b}{ }^{2}, \quad B^{a}{ }_{h}=B_{b}{ }^{j} g^{b a} g_{j h}, C^{x}{ }_{h}=C_{y}{ }^{j} g^{y x} g_{j h}$, and $K_{d c b}{ }^{a}$ and $K_{d c y}{ }^{x}$ are the curvature tensor of $M$ and that of the connection induced in the normal bundle of $M$ respectively.

From now on, we consider generic submanifolds of a Kaehlerian manifold $\tilde{M}$. Then we can put in each neighborhood

$$
\begin{gathered}
F_{\jmath}{ }^{h} B_{c}{ }^{\jmath}=f_{c}{ }^{a} B_{a}{ }^{h}-f_{c}{ }^{x} C_{x}{ }^{h}, \\
F_{\jmath}{ }^{h} C_{x}{ }^{\jmath}=f_{x}{ }^{a} B_{a}{ }^{h},
\end{gathered}
$$

where $f_{c}{ }^{a}$ is a tensor field of type (1.1) defined on $M, f_{c}{ }^{x}$ that of mixed type and $f_{x}{ }^{a}=f_{c}{ }^{y} g^{c a} g_{y x}$.

Applying $F$ to (1.11) and (1.12) respectively and using (1.1) and those equations, we can easily find

$$
\begin{gathered}
f_{c}^{e} f_{e}^{a}=-\delta_{c}^{a}+f_{c}^{x} f_{x}^{a}, \\
f_{c}^{e} f_{e}{ }^{x}=0, \quad f_{x}^{e} f_{e}^{a}=0, \\
f_{e} f_{y}{ }^{e}=\delta_{y}^{x} .
\end{gathered}
$$

Therefore, equations $(1.13) \sim(1.15)$ show that $M$ admits the so-called $f$-structure satisfying $f^{3}+f=0$ (cf. [6] and [7] etc.).

Using $F_{j i}=-F_{\imath \jmath}, F_{j i}=F_{\jmath}{ }^{h} g_{\imath h}$, we have from (1.11) and (1.12),

$$
f_{c b}=-f_{b c}, \quad f_{c x}=f_{x c},
$$

where we have put $f_{c b}=f_{c}{ }^{a} g_{b a}, f_{b x}=f_{b}{ }^{y} g_{y x}$ and $f_{x b}=f_{x}{ }^{a} g_{b a}$.

If we apply the operator $\nabla_{c}$ of the covariant differentiation to (1.11) and take account of (1.3), then we obtain 


$$
F_{\imath}{ }^{h} \nabla_{c} B_{b}{ }^{3}=\left(\nabla_{c} f_{b}{ }^{a}\right) B_{a}{ }^{h}+f_{b}^{a} \nabla_{c} B_{a}{ }^{h}-\left(\nabla_{c} f_{b}{ }^{x}\right) C_{x}{ }^{h}-f_{b}{ }^{x} \nabla_{c} C_{x}{ }^{h},
$$

or, substituting (1.6) and (1.7),

$$
\begin{gathered}
\nabla_{c} f_{b}{ }^{a}=h_{c b}{ }^{x} f_{x}{ }^{a}-h_{c}{ }^{a}{ }_{x} f_{b}{ }^{x}, \\
\nabla_{c} f_{b}{ }^{x}=h_{c e}{ }^{x} f_{b}{ }^{e} .
\end{gathered}
$$

By the same way we have from (1.12)

$$
\begin{aligned}
& \nabla_{c} f_{x}^{a}=h_{c e x} f^{a e}, \\
& f_{x}{ }^{e} h_{b e}{ }^{y}=h_{b}{ }^{e}{ }_{x} f_{e}^{y}
\end{aligned}
$$

with the help of (1.6) and (1.7).

We now assume that the ambient manifold $\tilde{M}$ is of constant holomorphic sectional curvature $c$. Then it is well known that its curvature tensor $K_{k j i}{ }^{h}$ has the form

$$
K_{k \jmath}{ }^{h}=\frac{c}{4}\left(\delta_{k}^{h} g_{j i}-\delta_{\jmath}^{h} g_{k i}+F_{k}{ }^{h} F_{j i}-F_{\jmath}{ }^{h} F_{k i}-2 F_{k \jmath} F_{\imath}{ }^{h}\right) .
$$

Therefore, substituting (1.21) into (1.8), (1.9) and (1.10), we can see that the equations of Gauss, Codazzi and Ricci are respectively given by

$$
\begin{aligned}
K_{d c b}{ }^{a}= & \frac{c}{4}\left(\delta_{d}^{a} g_{c b}-\delta_{c}^{a} g_{d b}+f_{d}{ }^{a} f_{c b}-f_{c}{ }^{a} f_{d b}-2 f_{d c} f_{b}{ }^{a}\right)+h_{d}{ }^{a}{ }_{x} h_{c b}{ }^{x}-h_{c}{ }^{a}{ }_{x} h_{d b}{ }^{x}, \\
& \nabla_{d} h_{c b}{ }^{x}-\nabla_{c} h_{d b}{ }^{x}=\frac{c}{4}\left(-f_{d}{ }^{x} f_{c b}+f_{c}{ }^{x} f_{d b}+2 f_{d c} f_{b}{ }^{x}\right), \\
& K_{d c y}{ }^{x}=\frac{c}{4}\left(f_{d}{ }^{x} f_{c y}-f_{c}{ }^{x} f_{d y}\right)+h_{d e}{ }^{x} h_{c}{ }^{e} y-h_{c e}{ }^{x} h_{d}{ }^{e} y
\end{aligned}
$$

We now consider a tensor field $S$ of type $(1,2)$ of the form

where

$$
S_{c b}{ }^{a}=[f, f]_{c b}{ }^{a}+\left(\nabla_{c} f_{b}{ }^{x}-\nabla_{b} f_{c}{ }^{x}\right) f_{x}{ }^{a},
$$

$$
[f, f]_{c b}{ }^{a}=f_{c}{ }^{e} \nabla_{e} f_{b}{ }^{a}-f_{b}{ }^{e} \nabla_{e} f_{c}{ }^{a}-\left(\nabla_{c} f_{b}{ }^{e}-\nabla_{b} f_{c}{ }^{e}\right) f_{e}{ }^{a}
$$

is the Nijenhuis tensor formed with $f_{c}{ }^{a}$.

Substituting (1.17) and (1.18) into this, we find

$$
S_{c b}{ }^{a}=\left(h_{c}{ }^{e} x f_{e}^{a}-f_{c}{ }^{e} h_{e}{ }^{a} x\right) f_{b}{ }^{x}-\left(h_{b}{ }^{e}{ }_{x} f_{e}^{a}-f_{b}{ }^{e} h_{e}{ }^{a} x\right) f_{c}{ }^{x} .
$$

The induced $f$-structure on $M$ is said to be normal if $S_{c b}{ }^{a}$ vanishes identically (cf. [4]).

The left hand side of (1.25) does not depend on the choice of the unit normals $C_{x}{ }^{h}$. Indeed, if we choose another set of mutually orthogonal unit normals ${ }^{\prime} C_{x}{ }^{h}$, then we have 


$$
{ }^{\prime} C_{x}{ }^{h}=\sigma_{x}{ }^{y} C_{y}{ }^{h},
$$

$\left(\sigma_{x}^{y}\right)$ being a special orthogonal matrix of degree $2 m-n$.

Defining the second fundamental tensor ${ }^{\prime} h_{c b}{ }^{x}$ with respect to ${ }^{\prime} C_{x}{ }^{h}$ by $\nabla_{c} B_{b}{ }^{h}$ $={ }^{\prime} h_{c b}{ }^{x} C_{x}{ }^{h}$, then we have from (1.6) and (1.26)

$$
{ }^{\prime} h_{c b}{ }^{x}=\sigma_{y}^{x} h_{c b}{ }^{y} .
$$

Also, we have from (1.11) and (1.26)

Consequently we have

$$
{ }^{\prime} f_{c}^{x}=\sigma_{y}^{x} f_{c}^{y}
$$

$$
\begin{aligned}
& \left(h_{c}{ }^{e} f_{e} f_{e}^{a}-f_{c}{ }^{e} h_{e}{ }^{a}{ }_{x}\right) f_{b}{ }^{x}-\left(h_{b}{ }^{e}{ }_{x} f_{e}{ }^{a}-f_{b}{ }^{e} h_{e}{ }^{a}{ }_{x}\right) f_{c}{ }^{x} \\
= & \left({ }^{\prime} h_{c}{ }^{e}{ }_{x} f_{e}{ }^{a}-f_{c}{ }^{e} h_{e}{ }^{a}{ }_{x}\right)^{\prime} f_{b}{ }^{x}-\left({ }^{\prime} h_{b}{ }^{e}{ }_{x} f_{e}{ }^{a}-f_{b}{ }^{\prime}{ }^{\prime} h_{e}{ }^{a}{ }_{x}\right)^{\prime} f_{c}{ }^{x}
\end{aligned}
$$

because of $\sigma_{z}{ }^{x} \sigma_{y x}=g_{z y}$. This shows that the condition imposed on $M$ is of intrinsic character.

Suppose that $S_{c b}{ }^{a}$ vanishes identically on $M$, we have from (1.25)

$$
\left(h_{c}^{e}{ }_{x} f_{e}^{a}-f_{c}^{e} h_{e}{ }^{a} x\right) f_{b}{ }^{x}-\left(h_{b}{ }^{e} f_{e}{ }^{a}-f_{b}^{e} h_{e}{ }^{a}{ }_{x}\right) f_{c}{ }^{x}=0,
$$

from which, transvecting $f^{c y}$,

$$
h_{a e^{y}} f_{b}^{e}+h_{b e} f_{a}^{e}=h_{c e x} f_{a}^{e} f^{c y} f_{b}{ }^{x},
$$

from which, taking the skew-symmetric part and then transvecting $f_{z}^{b}$, we get $h_{c e z} f_{a}{ }^{e} f^{c y}=0$.

Therefore, we obtain

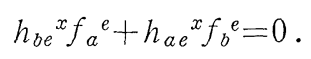

Hence we have

Proposition 1.1. Let $M$ be a generic submanifold of a Kaehlerian manifold $\tilde{M}$. In order for the f-structure induced on $M$ to be normal, it is necessary and sufficient that the second fundamental tensors $h_{c b}{ }^{x}$ and $f_{c}{ }^{a}$ commute.

\section{$\S 2$. Submersion $\tilde{\pi}: S^{2 m+1} \rightarrow C P^{m}$ and immersion $\imath: M \rightarrow C P^{m}$.}

Let $S^{2 m+1}(1)$ be the hypersphere $\left\{\left.\left(c^{1}, \cdots, c^{m+1}\right)|| c^{1}\right|^{2}+\cdots+\left|c^{m+1}\right|^{2}=1\right\}$ of radius 1 in the $(m+1)$-dimensional complex space $C^{m+1}$, which will be identified naturally with $R^{2(m+1)}$. The sphere $S^{2 m+1}(1)$ will be simply denoted by $S^{2 m+1}$. Let $\tilde{\pi}: S^{2 m+1} \rightarrow C P^{m}$ be the natural projection of $S^{2 m+1}$ onto a complex projective space $C P^{m}$ which is defined by the Hopf-fibration.

We consider a Riemannian submersion $\pi: \bar{M} \rightarrow M$ compatible with the Hopffibration $\tilde{\pi}: S^{2 m+1} \rightarrow C P^{m}$, where $M$ is a submanifold of codimension $p$ in $C P^{m}$ and 
$\bar{M}=\tilde{\pi}^{-1}(M)$ that of $S^{2 m+1}$. More precisely speaking, $\pi: \bar{M} \rightarrow M$ is a Riemannian submersion with totally geodesic fibres such that the following diagram is commutative :

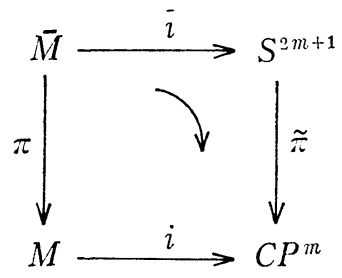

where $i: \bar{M} \rightarrow S^{2 m+1}$ and $\imath: M \rightarrow C P^{m}$ are certain isometric immersions.

Covering $S^{2 m+1}$ by a system of coordinate neighborhoods $\left\{\hat{U} ; y^{k}\right\}$ such that $\tilde{\pi}(\hat{U})=U$ are coordinate neighborhoods of $C P^{m}$ with local coordinate $\left(y^{h}\right)$, we represent the projection $\tilde{\pi}: S^{2 m+1} \rightarrow C P^{m}$ by

$$
y^{h}=y^{h}\left(y^{\kappa}\right)
$$

and put

$$
E_{\kappa}{ }^{h}=\partial_{\kappa} y^{h}, \quad \partial_{\kappa}=\partial / \partial y^{\kappa},
$$

the rank of the matrix $\left(E_{k}{ }^{h}\right)$ being always $2 m$.

Let's denote by $\tilde{\xi}^{\kappa}$ components of $\tilde{\xi}$ the unit Sasakian structure vector in $S^{2 m+1}$. Since the unit vector field $\tilde{\xi}$ is always tangent to the fibre $\tilde{\pi}^{-1}(\tilde{P}), \tilde{P} \in$ $C P^{m}$ everywhere, $E_{k}{ }^{h}$ and $\tilde{\xi}_{k}$ form a local coframe in $S^{2 m+1}$, where $\tilde{\xi}_{k}=g_{\mu \mu} \tilde{\xi}^{\prime \prime}$ and $g_{\kappa \mu}$ denote the Riemannian metric tensor of $S^{2 m+1}$. We denote by $\left\{E^{\kappa}{ }_{j}, \tilde{\xi}^{\kappa}\right\}$ the frame corresponding to the coframe $\left\{E_{\kappa}{ }^{h}, \tilde{\xi}_{k}\right\}$. We then have

$$
E_{\kappa}{ }^{\jmath} E^{\kappa}{ }_{\imath}=\delta_{\imath}^{\jmath}, \quad E_{\kappa}{ }^{\prime} \tilde{\xi}^{\kappa}=0, \quad \tilde{\xi}_{\kappa} E^{\kappa}{ }_{\imath}=0 .
$$

We now take coordinate neighborhoods $\left\{\bar{U} ; x^{\alpha}\right\}$ of $\bar{M}$ such that $\pi(\bar{U})=U$ are coordinate neighborhoods of $M$ with local coordinate $\left(x^{a}\right)$. Let the isometric immersions $i$ and $\imath$ be locally expressed by $y^{k}=y^{k}\left(x^{\alpha}\right)$ and $y^{h}=y^{h}\left(x^{a}\right)$ in terms of local coordinates $\left(x^{\alpha}\right)$ in $\bar{U}(\subset \bar{M})$ and $\left(x^{a}\right)$ in $U(\subset M)$ respectively. Then the commutativity $\tilde{\pi} \circ \bar{\imath}=\imath \supset \pi$ of the diagram implies

$$
y^{h}\left(x^{a}\left(x^{\alpha}\right)\right)=y^{h}\left(y^{\kappa}\left(x^{\alpha}\right)\right),
$$

where we expressed the submersion $\pi$ by $x^{a}=x^{a}\left(x^{\alpha}\right)$ locally, and hence

$$
B_{a}{ }^{\jmath} E_{\alpha}{ }^{a}=E_{\kappa}{ }^{\jmath} B_{\alpha}{ }^{\kappa},
$$

$B_{a}{ }^{\jmath}=\partial_{a} y^{\jmath}, B_{\alpha}{ }^{k}=\partial_{\alpha} y^{\kappa}$ and $E_{\alpha}{ }^{a}=\partial_{\alpha} x^{a}$.

For an arbitrary point $P \in M$ we choose unit normal vector fields $C_{x}{ }^{3}$ to $M$ defined in a neighborhood $U$ of $P$ in such a way that $\left\{B_{a}{ }^{j}, C_{x}{ }^{j}\right\}$ spans the tangent space of $C P^{m}$ at $i(P)$. Let $\bar{P}$ be an arbitrary point of the fibre $\pi^{-1}(P)$ over $P$ 
then the lifts $C_{x}{ }^{k}=C_{x}{ }^{j} E^{\kappa}$, of $C_{x}{ }^{j}$ are unit normal vector fields to $\bar{M}$ defined in the tubular neighborhood over $U$ because of (2.4). Since $\tilde{\xi}^{\kappa} E_{\kappa}{ }^{\jmath}=0$, we can represent by

$$
\tilde{\xi}^{\kappa}=\xi^{\alpha} B_{\alpha}{ }^{\kappa},
$$

where $\xi^{\alpha}$ is a local vector field in $\bar{M}$. Using (2.4) and (2.5), we find

$$
\xi_{\alpha} \xi^{\alpha}=1, \quad \xi^{\alpha} E_{\alpha}^{a}=0,
$$

where $\xi_{\alpha}=\xi^{\beta} g_{\beta \alpha}$ and $g_{\beta \alpha}$ is the Riemannian metric tensor of $\bar{M}$ induced from that of $S^{2 m+1}$. Therefore, $\left\{E_{\alpha}{ }^{a}, \xi_{\alpha}\right\}$ is a local coframe in $\bar{M}$ induced from that of $S^{2 m+1}$. Denote by $\left\{E_{a}^{\alpha}, \xi^{\alpha}\right\}$ the frame corresponding to this coframe $\left\{E_{\alpha}{ }^{a}, \xi_{a}\right\}$, we have

$$
E_{\alpha}{ }^{b} E^{\alpha}{ }_{a}=\delta_{a}^{b}, \quad \xi_{\alpha} E_{b}^{\alpha}=0,
$$

and consequently

$$
E^{\kappa}{ }_{j} B_{b}{ }^{\jmath}=B_{\alpha}{ }^{\kappa} E^{\alpha}{ }_{b}
$$

with the help of (2.4) and (2.6).

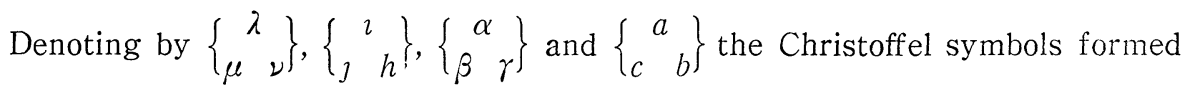
with the Riemannian metric $g_{\mu \lambda}, g_{j i}, g_{\beta \alpha}$ and $g_{b a}$ respectively, we put

$$
\begin{aligned}
& D_{\mu} E_{\lambda}{ }^{2}=\partial_{\mu} E_{\lambda}{ }^{2}-\left\{\begin{array}{c}
\kappa \\
\mu
\end{array}\right\} E_{\kappa}{ }^{2}+\left\{\begin{array}{c}
h \\
\jmath
\end{array} i^{\prime} E_{\mu}{ }^{\jmath} E_{\lambda}{ }^{h},\right.
\end{aligned}
$$

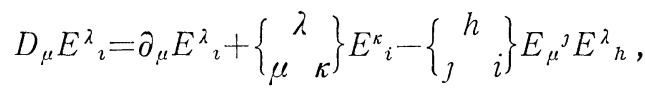

and

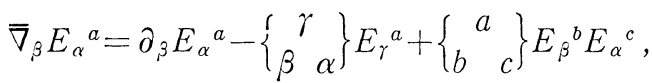

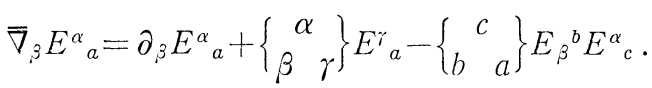

Since the metrics $g_{\lambda \mu}$ and $g_{\alpha \beta}$ are both invariant with respect to the submersions $\tilde{\pi}$ and $\pi$ respectively, the van der Waerden-Bortolotti covariant derivatives of $E_{\lambda}{ }^{\imath}, E^{\lambda}{ }_{\imath}$ and $E_{a}{ }^{a}, E^{\alpha}{ }_{a}$ are given by

$$
\begin{gathered}
\left\{\begin{array}{l}
D_{\mu} E_{\lambda}{ }^{\imath}=h_{\jmath}{ }^{2}\left(E_{\mu}{ }^{j} \tilde{\xi}_{\lambda}+\tilde{\xi}_{\mu} E_{\lambda}{ }^{j}\right), \\
D_{\mu} E^{\lambda}{ }_{\imath}=h_{j i} E_{\mu}{ }^{j} \tilde{\xi}^{\lambda}-h_{\imath}{ }^{j} \tilde{\xi}_{\mu} E^{\lambda}{ }_{j},
\end{array}\right. \\
\left\{\begin{array}{l}
\bar{\nabla}_{\beta} E_{\alpha}{ }^{a}=h_{b}{ }^{a}\left(E_{\beta}{ }^{b} \xi_{\alpha}+\xi_{\beta} E_{\alpha}{ }^{b}\right), \\
\bar{\nabla}_{\beta} E^{\alpha}{ }_{a}=h_{b a} E_{\beta^{b}}{ }^{b} \xi^{\alpha}-h_{a}{ }^{b} \xi_{\beta} E^{\alpha}{ }_{b}
\end{array}\right.
\end{gathered}
$$


respectively, where $h_{j}{ }^{2}=h_{j h} g^{i h}, h_{b}{ }^{a}=h_{b c} g^{c a}, h_{j \imath}$ and $h_{b a}$ being the structure tensors induced from the submersions $\tilde{\pi}$ and $\pi$ respectively (see Ishihara and Konishi [3]).

On the other hand the equations of Gauss and Weingarten for the immersion $i: \bar{M} \rightarrow S^{2 m+1}$ are given by

$$
\bar{\nabla}_{\beta} B_{\alpha}{ }^{k}=h_{\beta \alpha}{ }^{x} C_{x}{ }^{k}, \quad \bar{\nabla}_{\beta} C_{x}{ }^{k}=-h_{\beta}{ }^{\alpha}{ }_{x} B_{\alpha}{ }^{k},
$$

and those for the immersion $\imath: M \rightarrow C P^{m}$ by

$$
\nabla_{b} B_{a}{ }^{h}=h_{b a}{ }^{x} C_{x}{ }^{h}, \quad \nabla_{b} C_{x}{ }^{h}=-h_{b}{ }^{a}{ }_{x} B_{a}{ }^{h},
$$

where $h_{\hat{\hat{\beta}}}{ }^{\alpha}{ }_{x}=h_{\beta \gamma}{ }^{y} g^{\gamma \alpha} g_{y x}, h_{\beta \alpha}{ }^{x}$ and $h_{b a}{ }^{x}$ are the second fundamental tensors of $\bar{M}$ and $M$ with respect to the unit normals $C_{x}{ }^{k}$ and $C_{x}{ }^{h}$ respectively. Moreover, in such a case, (2.4) and (2.8) imply

$$
\nabla_{b}=E^{\alpha}{ }_{b} \bar{\nabla} .
$$

We now put $F_{\mu}{ }^{\lambda}=D_{\mu} \tilde{\xi}^{\lambda}$. Then we have by definition of the Sasakian structure

$$
F_{\mu}^{\lambda} F_{\kappa}^{\mu}=-\delta_{\kappa}^{\lambda}+\tilde{\xi}_{\kappa} \tilde{\xi}^{\lambda}, \quad F_{\mu}^{\lambda} \tilde{\xi}^{\mu}=0, \quad \tilde{\xi}_{\lambda} F_{\mu}^{\lambda}=0, \quad F_{\mu \lambda}+F_{\lambda \mu}=0
$$

and

$$
D_{\mu} F_{\lambda}{ }^{\kappa}=\tilde{\xi}_{\lambda} \delta_{\mu}^{\kappa}-\tilde{\xi}^{\kappa} g_{\mu \lambda}, \quad D_{\mu} \tilde{\xi}^{\lambda}=F_{\mu}{ }^{\lambda},
$$

where $F_{\mu \lambda}=g_{k \lambda} F_{\mu}{ }^{\lambda}$. Denoting by $\mathcal{L}$ the Lie differentiation with respect to the vector field $\tilde{\xi}$, we find

$$
\mathcal{L} F_{\mu}{ }^{\lambda}=0 .
$$

Putting in each neighborhood $U$

$$
F_{\jmath}{ }^{2}=F_{\mu}^{\lambda} E^{\mu}{ }_{\jmath} E_{\lambda}{ }^{\imath},
$$

we can see that $F_{j}{ }^{2}$ defines a global tensor field of the same type of $F_{\mu}{ }^{\lambda}$, which will be denoted by the same letter, with the help of (2.15), $\mathcal{L} E^{\lambda}{ }_{j}=0$ and $\mathcal{L} E_{\lambda^{2}}$ $=0$. Moreover, using (2.9), (2.14) and (2.16), we easily see

$$
F_{\jmath}{ }^{2}=-h_{\jmath}{ }^{2} \text {, }
$$

which satisfies

$$
F_{j}{ }^{h} F_{h}{ }^{2}=-\delta_{j}^{\imath} .
$$

Differentiating (2.6) covariantly along $C P^{m}$ and using (2.9) and (2.14), we have

$$
\nabla_{\imath} F_{j}^{h}=0
$$

where $\nabla$ denotes the projection of $D$. Hence the base space $C P^{m}$ admits a Kaehlerian structure $\left\{F_{\jmath}{ }^{2}, g_{j i}\right\}$ which is represented by the structure tensor $h_{j}{ }^{2}$ 
of the submersion $\tilde{\pi}: S^{2 m+1} \rightarrow C P^{m}$ defined by the Hopf-fibration.

Let's denote by $K_{\kappa \mu \nu}{ }^{\lambda}$ and $K_{k j i}{ }^{h}$ components of the curvature tensors of $\left(S^{2 m+1}, g_{\lambda \mu}\right)$ and $\left(C P^{m}, g_{j i}\right)$ respectively. Since the unit sphere is a space of constant curvature 1 , using the equations of co-Gauss, we have

$$
K_{k j i}{ }^{h}=K_{\kappa \mu \lambda}{ }^{\lambda} E^{\kappa}{ }_{k} E^{\mu}{ }_{j} E^{\nu}{ }_{2} E_{\lambda}{ }^{h}+h_{k}{ }^{h} h_{j \imath}-h_{\jmath}{ }^{h} h_{k i}-2 h_{k j} h_{\imath}{ }^{h}
$$

and together with (2.17)

$$
K_{k j i}{ }^{h}=\delta_{k}^{h} g_{j i}-\delta_{j}^{h} g_{k i}+F_{k}{ }^{h} F_{j i}-F_{\jmath}{ }^{h} F_{k i}-2 F_{k j} F_{\imath}{ }^{h} .
$$

Hence $C P^{m}$ is a Kaehlerian manifold with constant holomorphic sectional curvature 4 (cf. Ishihara and Konishi [3]).

Putting

$$
F_{\imath}{ }^{h} B_{b}{ }^{\imath}=f_{b}^{a} B_{a}{ }^{h}-f_{b}{ }^{x} C_{x}{ }^{h}, \quad F_{\imath}{ }^{h} C_{x}{ }^{\imath}=f_{x}{ }^{a} B_{a}{ }^{h},
$$

as already shown in $\S 1$, we can easily find the algebraic relations $(1.13) \sim(1.16)$ are the structure equations $(1.17) \sim(1.24)$ with $c=4$ which will be very useful. Now we put in each neighborhood $\bar{U}$ of $\bar{M}$

$$
f_{\beta}{ }^{\alpha}=f_{b}{ }^{a} E_{\beta}{ }^{b} E^{\alpha}{ }_{a} . \quad f_{x}{ }^{\alpha}=f_{x}{ }^{a} E^{\alpha}{ }_{a}, \quad f_{\alpha}{ }^{x}=f_{a}{ }^{x} E_{\alpha}{ }^{a},
$$

where here and in the sequel we denote the lifts of functions by the same letters as those the given functions. Then, using (2.4), (2.8), (2.20) and (2.21) and taking account of $C_{x}{ }^{\kappa}=C_{x}{ }^{j} E^{\kappa}{ }_{j}$, we obtain

$$
\begin{gathered}
F_{\mu}{ }^{\kappa} B_{\alpha}{ }^{\mu}=f_{\alpha}{ }^{\beta} B_{\beta}{ }^{\kappa}-f_{\alpha}{ }^{x} C_{x}{ }^{k}, \\
F_{\mu}{ }^{{ }} C_{x}{ }^{\mu}=f_{x}{ }^{\alpha} B_{\alpha}{ }^{k} .
\end{gathered}
$$

Transvecting $F_{\kappa}{ }^{\mu}$ to $(2.22)$ and (2.23) respectively and using (2.13), (2.22) and (2.23) in the usual way, we can easily obtain that

$$
\begin{gathered}
f_{\alpha}^{\gamma} f_{\gamma}^{\beta}=-\delta_{\beta}^{\alpha}+f_{x}^{\beta} f_{\alpha}^{x}+\xi_{\alpha} \xi^{\beta}, \\
f_{\alpha}^{\gamma} f_{\gamma}^{x}=0, \quad f_{x}^{\gamma} f_{\gamma}^{\alpha}=0, \\
f_{x}^{\gamma} f_{\gamma}^{y}=\delta_{x}^{y}, \\
f_{\alpha}^{\gamma} \xi_{\gamma}=0, \quad \xi^{\gamma} f_{\gamma}^{\alpha}=0, \\
f_{\gamma}^{x} \xi^{\gamma}=0, \quad \xi_{\gamma} f_{x}^{\gamma}=0, \\
f_{\beta \alpha}=-f_{\alpha \beta}, \quad f_{\alpha x}=f_{x \alpha},
\end{gathered}
$$

where we have put $f_{\beta \alpha}=f_{\beta}^{\gamma} g_{\gamma \alpha}, f_{\alpha x}=f_{\alpha}^{y} g_{y x}, f_{x \alpha}=f_{x}^{\beta} g_{\beta \alpha}$. Applying the operator $\bar{\nabla}_{\beta}=B_{\beta}{ }^{k} D_{\kappa}$ to (2.22) and (2.23) respectively and making use of (2.11), (2.14), (2.22) and (2.23), we also find

$$
\bar{\nabla}_{\gamma} f_{\beta}{ }^{\alpha}=-g_{\gamma \beta} \xi^{\alpha}+\delta_{\gamma}^{\alpha} \xi_{\beta}+h_{\gamma \beta}{ }^{x} f_{x}{ }^{a}-h_{\gamma}{ }^{\alpha}{ }_{x} f_{\beta}{ }^{x} .
$$




$$
\begin{gathered}
\bar{\nabla}_{\beta} f_{\alpha}{ }^{x}=h_{\beta \gamma}{ }^{x} f_{\alpha}^{\gamma}, \quad \bar{\nabla}_{\beta} f_{x}{ }^{\alpha}=-h_{\beta}{ }^{\gamma}{ }_{x} f_{\gamma}{ }^{\alpha}, \\
h_{\beta \alpha}{ }^{y} f_{x}{ }^{\alpha}=h_{\beta}{ }^{\alpha}{ }_{x} f_{\alpha}{ }^{y} .
\end{gathered}
$$

Also, applying the operator $\bar{\nabla}_{\beta}$ to (2.5) and taking account of (2.11) and (2.14), we have

$$
\bar{\nabla}_{\beta} \xi^{\alpha}=f_{\beta}{ }^{\alpha}, \quad \xi^{\alpha} h_{\beta \alpha}{ }^{x}=-f_{\beta}{ }^{x}, \quad h_{, \beta^{\alpha}}{ }_{x} \xi^{\beta}=-f_{x}{ }^{\alpha},
$$

which and (2.9) and (2.21) imply

$$
f_{b}{ }^{a}=-h_{b}{ }^{a} .
$$

Moreover, in such a submanifold $\bar{M}$, equations of Gauss, Codazzi and Ricc1 are respectively given by

$$
\begin{gathered}
K_{\delta \gamma \beta}{ }^{\alpha}=\delta_{\delta}^{\alpha} g_{\gamma \beta}-\delta_{\gamma}^{\alpha} g_{\delta \beta}+h_{\delta}{ }^{\alpha}{ }_{x} h_{\gamma \beta}{ }^{x}-h_{\gamma}{ }^{\alpha}{ }_{x} h_{\delta \beta}{ }^{x}, \\
\bar{\nabla}_{\gamma} h_{\beta \alpha}{ }^{x}-\bar{\nabla}_{\beta} h_{\gamma \alpha}{ }^{x}=0, \\
K_{\beta \alpha y}{ }^{x}=h_{\beta \gamma}{ }^{x} h_{\alpha}{ }^{\gamma}{ }-h_{\alpha \gamma}{ }^{x} h_{\beta^{\gamma}}{ }^{\gamma}{ }_{y},
\end{gathered}
$$

where $K_{\delta \gamma \beta}{ }^{\alpha}$ and $K_{\beta \alpha y}{ }^{\alpha}$ are components of the curvature tensor of $\bar{M}$ and those of the normal bundle of $\bar{M}$ respectively because the ambient manifold $S^{2 m+1}$ is a space of constant curvature 1 .

Now we apply the operator $\nabla_{b}=B_{b}{ }^{\jmath} \nabla_{\jmath}=E^{r}{ }_{b} \bar{\nabla}_{\gamma}$ to (2.4). Then, using (2.11) and (2.12), we have

$$
h_{b a}{ }^{x} C_{x}{ }^{\jmath} E_{\alpha}{ }^{a}+B_{a}{ }^{j} E^{\gamma}{ }_{b} \bar{\nabla}_{\gamma} E_{\alpha}{ }^{a}=B_{b}{ }^{j} E^{\mu}{ }_{i}\left(D_{\mu} E_{k}{ }^{j}\right) B_{\alpha}{ }^{k}+E_{\kappa}{ }^{\jmath} E^{\gamma}{ }_{b} h_{\gamma \alpha}{ }^{x} C_{x}{ }^{k},
$$

from which, taking account of (2.9), (2.10) and (2.34),

$$
h_{b a}{ }^{x} C_{x}{ }^{j} E_{\alpha}{ }^{a}-f_{b}{ }^{a} B_{a}{ }^{j} \xi_{\alpha}=-F_{\imath}{ }^{\jmath} B_{b}{ }^{j} \xi_{\alpha}+\left(h_{\beta \alpha}{ }^{x} E^{\beta}{ }_{b}\right) C_{x}{ }^{j},
$$

or, using (2.20),

$$
h_{\beta \alpha}{ }^{x} E^{\beta}{ }_{b}=h_{b a}{ }^{x} E_{\alpha}{ }^{a}-f_{b}{ }^{x} \xi_{\alpha} .
$$

Transvecting (2.38) with $E_{\gamma}{ }^{b}$ and changing the index $\gamma$ with $\beta$, we get

$$
h_{\beta \alpha}{ }^{x}=h_{b a}{ }^{x} E_{,{ }^{b}} E_{\alpha}{ }^{a}-f_{\beta}{ }^{x} \xi_{\alpha}-\xi_{\beta} f_{\alpha}{ }^{x}
$$

with the help of (2.21) and (2.33).

Thus we have

LEMma 2.1. The mean curvature of $\bar{M}$ is the same as that of $M$.

Therefore, from now on, we write $h_{e} e^{e x}$ and $h_{\alpha}{ }^{\alpha x}$ as the same letter $h^{x}$ Moreover, the mean curvature vector $\bar{M}$ is given by 


$$
H^{\kappa}=\frac{1}{n+1} h^{x} C_{x}{ }^{\kappa} .
$$

The mean curvature vector $H$ is said to be parallel in the normal bundle of $\bar{M}$ if $\bar{\nabla}_{\beta} h^{x}=0$. Hence, as a direct consequence of Lemma 2.1 , we have

LEMMA 2.2. The mean curvature vector of $\bar{M}$ is parallel in the normal bundle of $\bar{M}$ if and only if the mean curvature vector of $M$ is parallel in the normal bundle of $M$.

Transvecting $h_{\gamma}{ }^{\alpha}{ }_{y}$ to (2.39) and using (2.21), (2.26), (2.28) and (2.38) imply

$$
h_{\beta \gamma}{ }^{x} h_{\alpha}^{\gamma}{ }_{y}=\left(h_{b a}{ }^{x} h_{c}^{a}{ }_{y}+f_{b}{ }^{x} f_{c y}\right) E_{\beta}{ }^{b} E_{\alpha}{ }^{c}-h_{b a}{ }^{x} f_{y}{ }^{a} E_{\beta}{ }^{b} \xi_{\alpha}
$$

which and (1.20) gives

$$
-h_{b}{ }^{a}{ }_{y} f_{a}{ }^{x} \xi_{\beta} E_{\alpha}{ }^{b}+\left(f_{\gamma}{ }^{x} f_{y}^{\gamma}\right) \xi_{\beta} \xi_{\alpha}
$$

$$
h_{\beta \gamma}{ }^{x} h_{\alpha}^{\gamma}{ }_{y}-h_{\alpha \gamma}{ }^{x} h_{\beta}^{\gamma}{ }_{y}=\left(f_{d}^{x} f_{c y}-f_{c}^{x} f_{d y}+h_{d e}{ }^{x} h_{c}{ }^{e} y-h_{c e}{ }^{x} h_{d y}{ }^{e}\right) E_{\beta}{ }^{d} E_{\alpha}{ }^{c},
$$

that is,

Thus we obtain

$$
K_{\beta \alpha y}{ }^{x}=K_{d c y}^{x} E_{\beta}^{d} E_{\alpha}{ }^{c} .
$$

LEMMA 2.3. In order that the connection in the normal bundle of $\bar{M}$ in $S^{2 m+1}$ is flat, it is necessary and sufficient that the connection in the normal bundle of $M$ in $C P^{m}$ is flat.

\section{$\S 3$. Generic submanifolds of a complex projective space admitting the normal $f$-structure.}

In this section we assume that the $f$-structure induced on $M$ in $C P^{m}$ is normal and the normal connection of $M$ is flat, that is,

$$
h_{b e}^{x} f_{a}^{e}+h_{a e^{x}} f_{b}^{e}=0
$$

and

$$
f_{d}^{x} f_{c y}-f_{c}^{x} f_{d y}+h_{d e}{ }^{x} h_{c}^{e} y-h_{c e} h_{d y}^{e}=0
$$

with the help of (1.24) with $c=4$.

Transvecting (3.1) with $f_{c}{ }^{a}$ and making use of (1.13), we obtain

$$
h_{c b}{ }^{x}-\left(h_{b e}{ }^{x} f_{y}^{e}\right) f_{c}^{y}+h_{d e}{ }^{x} f_{c}^{d} f_{b}^{e}=0,
$$

from which, taking the skew-symmetric part with respect to $c$ and $b$,

$$
\left(h_{c e}{ }^{x} f_{y}^{e}\right) f_{b}^{y}-\left(h_{b e}{ }^{x} f_{y}{ }^{e}\right) f_{c}^{y}=0 .
$$

Transvection $f_{z}^{c}$ gives 


$$
h_{b e}{ }^{x} f_{y}^{e}=P_{y z}^{x} f_{b}^{z},
$$

where we have put

$$
P_{y z}{ }^{x}=h_{c b}{ }^{x} f_{y}{ }^{c} f_{z}^{b} \text {. }
$$

Putting $P_{y z x}=P_{y z}{ }^{w} g_{w x}$, we see that $P_{y z x}$ is symmetric for all indices because of (1.20) and (3.4). Also, transvecting (3.2) with $f_{z}^{c}$ and using (3.3), we find

or, using (1.15),

$$
P_{z y}^{u} P_{u w}^{x} f_{d}^{w}-P_{z u}^{x} P_{y w}^{u} f_{d}^{w}=\delta_{z}^{x} f_{d y}-f_{d}^{x} g_{y z},
$$

$$
P_{z y}{ }^{u} P_{u w}{ }^{x}-P_{z u}^{x} P_{y w}^{u}=\delta_{z}^{x} g_{y w}-\delta_{w}^{x} g_{y z},
$$

from which,

$$
P_{z u}^{x} P_{y x}^{u}=P_{x} P_{y z}^{x}+(p-1) g_{y z},
$$

where we have put

$$
P^{x}=g^{y z} P_{y z}^{x}
$$

Now we prove

LEMMA 3.1. Let $M$ be an n-dimensional generic submanifold of $C P^{m}$ with flat normal connection. If the $f$-structure induced on $M$ is normal, then we have

$$
h_{\beta \alpha}{ }^{x} h_{\gamma}^{\alpha}=P_{y z}^{x} h_{\beta \gamma}{ }^{2}+g_{\beta \gamma} \delta_{y}^{x} .
$$

Proof. Differentiating (3.3) covariantly along $M$ and then taking the skewsymmetric part of what obtained thus, we have

$$
\begin{aligned}
& \left(\nabla_{c} h_{b e}{ }^{x}-\nabla_{b} h_{c e}{ }^{x}\right) f_{y}{ }^{e}+h_{c e}{ }^{x} h_{b}{ }^{a}{ }_{y} f_{a}{ }^{e}-h_{b e}{ }^{x} h_{c}{ }^{a}{ }_{y} f_{a}{ }^{e} \\
& =\left(\nabla_{c} P_{y z}{ }^{x}\right) f_{b}{ }^{z}-\left(\nabla_{b} P_{y z}{ }^{x}\right) f_{c}{ }^{z}+P_{y z}{ }^{x} h_{c e}{ }^{z} f_{b}{ }^{e}-P_{y z}{ }^{x} h_{b e}{ }^{z} f_{c}{ }^{e}
\end{aligned}
$$

with the help of (1.18). Substituting (1.23) with $c=4$ and making use of (3.2), then it must be that

$$
2 f_{c b} \delta_{y}^{x}+2 h_{c e}{ }^{x} h_{a}{ }_{y} f_{b}{ }^{a}=\left(\nabla_{c} P_{y z}{ }^{x}\right) f_{b}^{z}-\left(\nabla_{b} P_{y z}{ }^{x}\right) f_{c}^{z}+2 P_{y z}{ }^{x} h_{c e}{ }^{z} f_{b}^{e}
$$

with the help of (3.1). Transvecting (3.9) with $f_{w}{ }^{b}$ and using (1.14) and (1.15),

$$
\nabla_{c} P_{y w}^{x}=f_{w}^{b}\left(\nabla_{b} P_{y z}^{x}\right) f_{c}^{z} .
$$

Therefore (3.9) reduces to

$$
f_{c b} \delta_{y}^{x}+h_{c e}{ }^{x} h_{a}^{e}{ }_{y} f_{b}^{a}=P_{y z}{ }^{x} h_{c e}{ }^{z} f_{b}^{e}
$$

with the help of $P_{y z}{ }^{x}=P_{z y}{ }^{x}$. Transvecting (3.10) with $f_{d}^{b}$, we have

$$
\left(g_{c d}-f_{c}^{z} f_{d z}\right) \delta_{y}^{x}-h_{c e}{ }^{x} h_{d}{ }^{e} y+h_{c e}{ }^{x} h_{a}{ }^{e} f_{d}{ }^{z} f_{z}^{a}=-P_{y z}{ }^{x} h_{c d}{ }^{z}+P_{y z}{ }^{x} h_{c e}{ }^{z} f_{d}{ }^{w} f_{w}{ }^{e} .
$$


Taking account of (3.3) and (3.5), then (3.11) gives

$$
\begin{aligned}
& \left(g_{c d}-f_{c}^{z} f_{d z}\right) \delta_{y}^{x}-h_{c e}{ }^{x} h_{d y}{ }^{e}+P_{w u}{ }^{x} P_{z y}{ }^{w} f_{d}{ }^{z} f_{c}{ }^{u} \\
& \quad=-P_{y z}{ }^{x} h_{c d}{ }^{z}+f_{c}^{x} f_{d y}-\delta_{y}^{x} f_{c}^{z} f_{z d}+P_{w u}{ }^{x} P_{z y}{ }^{w} f_{d}{ }^{z} f_{c}{ }^{u} .
\end{aligned}
$$

Consequently, we obtain

$$
h_{c e}{ }^{x} h_{b}{ }^{e}=P_{y z}{ }^{x} h_{c b}{ }^{z}+g_{c b} \delta_{y}^{x}-f_{c}^{x} f_{b y} .
$$

Substitution (3.12) into (2.41) yields

$$
h_{\beta \alpha}{ }^{x} h_{\gamma}{ }^{\alpha}=P_{y z}{ }^{x} h_{\beta \gamma}{ }^{2}+g_{\beta \gamma} \delta_{y}^{x}
$$

with the help of (2.21), (2.39) and (3.3). This completes the proof of our lemma.

On the other hand, by the straightforward computation we get

$$
h_{\beta \alpha}{ }^{x} f_{\gamma}^{\alpha}+h_{\gamma \alpha}{ }^{x} f_{\beta}^{\alpha}=0
$$

with the help of (2.24), (2.25), (2.26), (2.27), (2.39) and (3.1). Transvection (2.39) with $f_{y}{ }^{\alpha}$ gives

$$
h_{\beta \alpha}{ }^{x} f_{y}^{\alpha}=P_{y z}^{x} f_{\beta}^{z}-\delta_{y}^{x} \xi_{\beta}
$$

with the help of (2.21) and (3.3), from which, transvecting $f_{w}{ }^{\beta}$, we find

$$
P_{y z}^{x}=h_{\beta \alpha}{ }^{x} f_{y}^{\beta} f_{z}^{\alpha},
$$

or, transvecting $g^{y z}$,

$$
P^{x}=h_{\beta \alpha}^{x} f_{y}^{\beta} f^{y \alpha} .
$$

Now, differentiating (3.13) covariantly and using (2.31), we find

$$
\begin{gathered}
\left(\bar{\nabla}_{\delta} h_{\beta \alpha}{ }^{x}\right) f_{\gamma}{ }^{\alpha}+h_{\beta \alpha}{ }^{x}\left(-g_{\delta \gamma} \xi^{\alpha}+\delta_{\delta}^{\alpha} \xi_{\gamma}+h_{\delta \gamma}{ }^{y} f_{y}{ }^{\alpha}-h_{\delta}{ }^{\alpha}{ }_{y} f_{\gamma}{ }^{y}\right)+\left(\bar{\nabla}_{\delta} h_{\gamma \alpha}{ }^{x}\right) f_{\beta}{ }^{\alpha} \\
+h_{\gamma \alpha}{ }^{x}\left(-g_{\delta \beta} \xi^{\alpha}+\delta_{\delta}^{\alpha} \xi_{\beta}+h_{\delta \beta}{ }^{y} f_{y}{ }^{\alpha}-h_{\delta}{ }^{\alpha}{ }_{y} f_{\beta}{ }^{y}\right)=0,
\end{gathered}
$$

or, using (2.33), (3.8) and (3.14),

$$
\left(\bar{\nabla}_{\delta} h_{\beta \alpha}{ }^{x}\right) f_{\gamma}{ }^{\alpha}+\left(\bar{\nabla}_{\delta} h_{\alpha \gamma}{ }^{x}\right) f_{\beta}{ }^{\alpha}=0,
$$

from which, taking the skew-symmetric part with respect to the indices $\delta$ and $\beta$,

$$
\left(\bar{\nabla}_{\delta} h_{\gamma \alpha}{ }^{x}\right) f_{\beta}{ }^{\alpha}-\left(\bar{\nabla}_{\beta} h_{\gamma \alpha}{ }^{x}\right) f_{\delta}^{\alpha}=0
$$

since the ambient manifold $S^{2 m+1}$ is a space of constant curvature 1 . Hence the last two equations imply $\left(\bar{\nabla}_{\gamma} h_{\beta \alpha}{ }^{x}\right) f_{\delta}^{\alpha}=0$, from which, transvecting $f_{\varepsilon}^{\delta}$, we find

$$
\bar{\nabla}_{\gamma} h_{\beta \varepsilon}{ }^{x}=\left(\bar{\nabla}_{\gamma} h_{\beta \alpha}{ }^{x}\right) f_{\varepsilon}{ }^{y} f_{y}{ }^{\alpha}+\left(\bar{\nabla}_{\gamma} h_{\beta \alpha}{ }^{x}\right) \xi_{\varepsilon} \xi^{\alpha}
$$

by virtue of (2.24). Transvection this equation with $g^{\beta \varepsilon}$ gives 


$$
\bar{\nabla}_{\gamma} h^{x}=\left(\bar{\nabla}_{\gamma} h_{\beta \alpha}{ }^{x}\right) f^{\beta y} f_{y}{ }^{\alpha}+\left(\bar{\nabla}_{\gamma} h_{\beta \alpha}{ }^{x}\right) \xi^{\beta} \xi^{\alpha} .
$$

By the straightforward computation, we find

$$
\left(\bar{\nabla}_{\gamma} h_{\beta \alpha}{ }^{x}\right) \xi^{\beta} \xi^{\alpha}=0
$$

because of (2.28) and (2.33). Consequently, we have

$$
\bar{\nabla}_{\gamma} h^{x}=\left(\bar{\nabla}_{\gamma} h_{\beta \alpha}{ }^{x}\right) f^{\beta y} f_{y}{ }^{\alpha} .
$$

If we differentiate (3.16) covariantly and use (3.17), then we obtain

$$
\bar{\nabla}_{\gamma} P^{x}=\bar{\nabla}_{\gamma} h^{x}+h_{\beta \alpha}{ }^{x}\left(\bar{\nabla}_{\gamma} f^{\beta y}\right) f_{y}{ }^{\alpha}+h_{\beta \alpha}{ }^{x} f^{\beta y} \bar{\nabla}_{\gamma} f_{y}{ }^{\alpha},
$$

or, substitute (2.25) and make use of (3.14), we get

Thus we have

$$
\bar{\nabla}_{\gamma} P^{x}=\bar{\nabla}_{\gamma} h^{x} .
$$

LEMMA 3.2. Under the same assumptions as those stated in Lemma 3.1, we have

$$
\bar{\nabla}_{\gamma} P^{x}=\bar{\nabla}_{\gamma} h^{x} .
$$

Next, we prove

LEMMA 3.3. Under the same assumptions as those stated in Lemma 3.1, we have

$$
\frac{1}{2} \Delta\left(h_{\beta \alpha}{ }^{x} h^{\beta \alpha}{ }_{x}\right)=\left(\bar{\nabla}_{\beta} \bar{\nabla}_{\alpha} h^{x}\right) h^{\beta \alpha}{ }_{x}+\left\|\bar{\nabla}_{\gamma} h_{\beta \alpha}{ }^{2}\right\|^{2},
$$

where $\Delta$ is the Laplacian given by $\Delta=g^{\gamma \beta} \bar{\nabla}_{\gamma} \bar{\nabla}_{\beta}$.

Proof. From the Ricci identity, we have

$$
\bar{\nabla} r \bar{\nabla}_{\gamma} h_{\beta \alpha}{ }^{x}-\bar{\nabla}_{\beta} \bar{\nabla}_{\alpha} h^{x}=K_{\beta \gamma} h_{\alpha}^{\gamma x}-K_{\delta \beta \alpha \gamma} h^{\gamma \delta x}
$$

with the help of (2.36), where $K_{\beta r}$ is the Ricci tensor given by

$$
K_{\beta \gamma}=n g_{\beta \gamma}+h^{x} h_{\beta \gamma x}-h_{\beta \alpha}{ }^{x} h_{\gamma}{ }^{\alpha}{ }
$$

by virtue of (2.35). If we transvect (3.20) with $h^{\beta \alpha}{ }_{x}$ and take account of (2.37), (3.8) and (3.21), then we find

$$
\left(\bar{\nabla} r \bar{\nabla}_{\gamma} h_{\beta \alpha}{ }^{x}\right) h^{\beta \alpha}{ }_{x}-\left(\bar{\nabla}_{\beta} \bar{\nabla}_{\alpha} h^{x}\right) h^{\beta \alpha}{ }_{x}=0
$$

with the help of (3.6). Therefore, we have the Laplacian of the length of the second fundamental tensors $h_{\beta \alpha}{ }^{x}$ as follows:

$$
\frac{1}{2} \Delta\left(h_{\beta \alpha}{ }^{x} h_{x}^{\beta \alpha}\right)=\left(\bar{\nabla}_{\beta} \bar{\nabla}_{\alpha} h^{x}\right) h^{\beta \alpha}{ }_{x}+\left\|\bar{\nabla}_{\gamma} h_{\beta \alpha}{ }^{x}\right\|^{2} .
$$


Thus we complete the proof of this lemma.

If the mean curvature vector of $M$ is parallel in the normal bundle, then it follows that the mean curvature vector of $\bar{M}$ is also parallel in the normal bundle by means of Lemma 2.2. Therefore, $h_{\beta \alpha}{ }^{x} h^{\beta \alpha}{ }_{x}=h_{x} P^{x}+(n+1) p$, which is induced from (3.8), is a constant along $\bar{M}$ because of (3.18). Hence (3.19) reduces to $\bar{\nabla}_{\gamma} h_{\beta \alpha}{ }^{x}=0$. Since $\bar{M}$ is of essential codimension $2 m-n$ and does not admit umbilical sections because of (3.14), combining with Theorem $\mathrm{A}$ is $\S 0$, we have

THEOREM 3.4. Let $M$ be an n-dimensional complete generic submanifold of a complex projective space $C P^{m}$ with flat normal connection. If the f-structure induced on $M$ is normal and if the mean curvature vector of $M$ is parallel in the normal bundle, then $M$ is of the form

$$
\begin{aligned}
& \tilde{\pi}\left(S^{p_{1}}\left(r_{1}\right) \times \cdots \times S^{p_{N}}\left(r_{N}\right)\right), p_{1}, \cdots, p_{N} \text { are odd numbers } \geqq 1, \\
& p_{1}+p_{2}+\cdots+p_{N}=n+1, r_{1}^{2}+r_{2}^{2}+\cdots+r_{N}^{2}=1, N=2 m-n+1,
\end{aligned}
$$

where $S^{p_{i}}\left(r_{2}\right)$ is a $p_{i}$-dimensional sphere with radius $r_{\imath}$.

\section{REFERENCES}

[1] CHEN, B. Y., Geometry of submanifolds, Marcel Dekker Inc., N. Y., 1973.

[2] H. B. Lawson, Jr., Rigidity theorems in rank 1 symmetric spaces. J. Diff. Geo., 4 (1970), 349-359.

[3] Ishihara, S. AND M. Konishi, Differential geometry of fibred spaces, Publication of study group of geometry, Vol. 8, Tokyo. 1973.

[4] KI, U-HANG AND J. S. PAK, Generic submanifolds of an even-dimensional Euclidean space, to appear in J. Diff. Geo..

[5] MaEdA, Y., On real hypersurfaces of a complex projective space, J. Math. Soc. of Japan, 28 (1976), 529-540.

[6] Nakagawa, H., On framed $f$-manifolds, Kōdai Math. Sem. Rep., 18 (1966), 293306.

[7] NAKAGAwa, H., The $f$-structure induced on submanifolds of complex and almost complex spaces, Kōdai Math. Sem. Rep., 18 (1966), 120-160.

[8] OKumura, M., On some real hypersurfaces of a complex projective space, Transactions of AMS., 212 (1975), 355-364.

[9] Okumura, M., Submanifolds of real codimension of a complex projective space, Atti della Accademia Nazionale dei Lince1, 4 (1975), 544-555.

[10] PAK, J.S., Note on anti-holomorphic submanifolds of real codimension of a complex projective space, to appear in Kyungpook Math. J.

[11] YANO, K. AND S. IshiHARA, Submanifolds with parallel mean curvature vector, J. Diff. Geo., 6 (1971), 95-118.

[12] Yano, K. And M. Kon, Generic submanifolds, to appear in Annali di Mat.

KYUNGPOOK UNIVERSITY

TAEgu, Korea 